\title{
Entrevista com a professora de filosofia: Marisa Berttolini
}

\author{
Por: Elisete M. Tomazetti
}

Elisete: Gostaríamos de iniciar perguntando sobre sua trajetória intelectual e profissional, sua formação e sua relação com a Educação e, em especial, com o ensino de Filosofia. Pode nos falar brevemente sobre isso?

Marisa: Soy profesora de Filosofía, egresada del Instituto de Profesores Artigas, Institución que en Uruguay forma docentes para la Educación Media en todas las disciplinas. Trabajé durante muchos años en distintos liceos públicos y privados. Desde la década de los 90 asumí cursos de Didáctica de la Filosofía y me fui especializando en esta orientación. En el 2000 accedí al cargo de Inspectora de Filosofía en la Educación Media. Desde esta función estuve en diálogo permanente con los docentes, impulsando líneas pedagógicas y filosóficas orientadas a potenciar el lugar de la asignatura para estimular el pensamiento crítico y creativo de los estudiantes. Participé también, junto a varios colegas, en la reformulación de los Programas de Filosofía. En los últimos años integro un equipo de investigación, junto con la Profesora Isabel Gonzáles y el Profesor Mauricio Langon, equipo con el que estamos indagando sobre la enseñanza de la Filosofía. Durante esta trayectoria, mi concepción sobre la educación filosófica tuvo cambios significativos.

Elisete: Como você descreveria a situação atual do ensino de filosofia nas escolas do Uruguai? Qual a perspectiva didática que orienta as práticas dos professores de Filosofia?

Marisa: En el Uruguay la asignatura Filosofía está presente en los 3 últimos años de Educación Media con una carga horaria de 4 o 3 horas semanales en todas las orientaciones de Bachillerato. También aparece en el currículo de Formación Docente para maestros de primaria y profesores de secundaria de todas las disciplinas en la modalidad Filosofía de la Educación y Epistemología. 
Entre fines de los 80 y principios de los 90 comienza a profundizarse en nuestro país la reflexión sobre el sentido de la enseñanza de la Filosofía, y se establecen vínculos con movimientos análogos en otros países de la región. Sin duda el contexto de salida de las dictaduras habilita y exige repensar la función de la educación en las nuevas coyunturas democráticas y en alerta sobre el riesgo del retorno de regímenes totalitarios y represores.

En relación a la enseñanza de la Filosofía una convicción fundamental es que la reflexión debe hacerse desde categorías filosóficas evitando toda reducción a enfoques tecnicistas. Es decir la didáctica de la Filosofía es a la vez un pensar filosófico.

En este contexto se instala entre los docentes un debate que se puede resumir en la dicotomía entre "enseñar Filosofía" o "enseñar a Filosofar", discusión que, en nuestra perspectiva, coloca una falacia de falsa oposición entre posibilidades que poco a poco se van comprendiendo como complementarias.

Cuando proponemos, por ejemplo, estructurar la clase de Filosofía centrada en problemas, esto no supone despojarla de contenidos. Por el contrario, debe haber un lugar, y central para transmitir la producción filosófica tal como se ha dado históricamente y para la lectura fermental de textos filosóficos. La experiencia del alumno, sus intereses, sus vivencias más fuertes, sus circunstancias, deben ser siempre el punto de partida y un referente constante de la propuesta del docente. Pero nunca el eje único sobre el cual desarrollar la clase. La tarea nuestra es, muchas veces, "violentar" la espontaneidad del alumno, proponerle tareas que no le resultan gratificantes de inmediato, exigirle una actitud intelectual para la que no siempre está predispuesto. Lo que llamamos "actitud espontánea del alumno" no siempre supone autonomía. .Es a menudo reflejo de la ideología hegemónica del contexto cultural en que se mueve. Respetar absolutamente su espontaneidad, puede tener como resultado escamotear su libertad. Creemos, entonces, que hay algunos ejes fundamentales sobre los cuales sustentar el pensamiento filosófico, también en clase: precisión conceptual, información, y rigor en la argumentación. Es deseable tener en cuenta diversas franjas de actividad a desarrollar en el aula: problematización, información, reflexión, discusión. Estas franjas complementarias 
posibilitarán el desarrollo en los alumnos de ciertas capacidades esenciales al pensamiento filosófico. Por ejemplo, formular buenas preguntas, enunciar con precisión las propias ideas, dar razones para justificar las afirmaciones, descubrir los supuestos o premisas implícitas en las opiniones, percibir las implicaciones o consecuencias que derivan de una tesis, explicitar los criterios sobre los que se apoyan los juicios.

Elisete: Como se dá a formação dos professores de Filosofia para atuação no ensino médio?

Marisa: En el Uruguay, la formación de docentes para la Educación Media, se desarrolla durante 4 años. En el currículo se incluyen asignaturas específicas de cada disciplina y otras de formación pedagógica. El eje articulador esencial es el espacio de Didáctica, que incluye una instancia teórica con el docente especializado y una instancia práctica a lo largo de 3 años. Durante 2 años el estudiante participa, a lo largo de todo el curso, como observador en una clase de otro profesor adscriptor que funciona como "tutor" y donde hace algunas intervenciones y en el último año de su formación cada estudiante tiene un grupo a su cargo durante todo el año, con la supervisión del profesor de didáctica.

Casi en su totalidad los cursos de Filosofía en los distintos niveles cuentan con docentes egresados de los Institutos de formación docente. O sea, profesores que poseen formación disciplinar y didáctica.

Elisete: Há no Uruguai uma política pública para os livros didáticos? Quais são os materiais didáticos mais utilizados pelos professores em suas aulas?

Marisa: Estrictamente no hay una política pública para los libros didácticos. En realidad muchos docentes nos resistimos a que haya Manuales oficiales y uniformes para la enseñanza de la Filosofía.

Los programas están pensados para que cada profesor se transforme em constructor de su proprio curso (sólo ateniéndose a los lineamientos generales). Sin 
embargo, se producen con frecuencia textos pensados para orientar las clases. Muchos colegas han tomado la iniciativa y han aportado en este sentido . Yo misma participé en producciones colectivas que se publicaron con el título de Materiales para la construcción de cursos de Filosofía. Propuestas didácticas para la problematización y la discusión. Se editaron 2 libros: "¿Qué es Filosofía?"AZ Editora. Montevideo. 1997, en coautoría con el Prof. Langon y la Profa. Quintela. Y Diversidad Cultural e Interculturalidad. Ediciones Novedades Educativas. Buenos Aires. 2009, en coautoría con Mauricio Langon.

Pero ninguno de estos libros tiene carácter prescriptivo ni oficial. Muchos de los textos cuentan con el aval de la Inspección pero se trata solamente de sugerencias.

La contrapartida de esta libertad creadora, es, en muchos casos, el uso por parte de los profesores de fragmentos de textos fotocopiados de muy diversos autores que distribuyen entre los alumnos y sobre los cuales organizan las clases. En algunos casos los fragmentos circulan sin una precisa referencia bibliográfica y en forma dispersa, lo que afecta el nivel académico de los materiales.

Elisete: Os jovens alunos uruguaios se interessam por filosofia? Como é a recepção da filosofia por eles? Como você descreveria a relação dos jovens alunos com a leitura e a escrita filosófica? As tecnologias da informação e comunicação, os meios digitais, são utilizados nas aulas de Filosofia?

Marisa: La actitud de los jóvenes frente a la educación filosófica es ambivalente. Por un lado, los hábitos internalizados para aprender memorísticamente ciertos contenidos y la exigencia de reproducirlos em las instancias de evaluación, provocan un profundo desconcierto frente a propuestas metodológicas que aspiran a la participaicón creativa y al desarrollo del pensamiento autónomo en las clases de Filosofia. Pero muy pronto muchos alumnos reconocen el valor de estos espacios, visualizan la significación del pensamiento filosófico y así se alcanzan logros interesantes. 
Elisete: Há programas de formação continuada para os professores de Filosofia? Quem os promove? Quais suas características?

Marisa: Si, hay propuestas de formación permanente para los docentes de Filosofía. Son promovidos por la Inspección de Filosofía, el Departamento de Filosofía del Instituto de Profesores Artigas y/o por la Asociación Filosófica del Uruguay.

Resultan aportes muy valiosos y reconocidos por los profesores. En general son cursos o talleres de profundización disciplinar y sobre didáctica de la filosofía. Estos encuentros no sólo constituyen instancias de actualización académica sino la ocasión de intercambios fermentales entre colegas, intercambios vitales para discutir y mejorar las prácticas cotidianas.

Elisete: Poderias nos dizer de suas pesquisas e estudos sobre ensino de Filosofia? Que filósofos e filosofias lhe oferecem referências para seu pensamento sobre essa temática?

Marisa: El equipo que integro ( junto con los profesores Isabel González y Mauricio Langon) realizó tres investigaciones:

Escenarios de La educación filosófica. Prácticas y discursos: Exploración, reconstrucción y posibilidades de transformación en el marco de la asignatura Filosofía en el nivel medio del sistema educativo uruguayo. 2008

Tensiones en la enseñanza de la filosofía. Perspectivas para pensar prácticas y discursos. 2010

Argumentación: un aporte filosófico a la educación, 2015.

Las dos primeras investigaciones son complementarias. La primera desarrolló el trabajo de campo, observando y registrando clases de Filosofía y la segunda profundizó en la interpretación del material recopilado. Los objetivos fueron: estudiar el aula de filosofía; analizar prácticas de enseñanza y aprendizaje; determinar las características de una buena clase filosófica; analizar las posibilidades de 
transferencia de las habilidades de pensamiento filosófico a la enseñanza de otras disciplinas, en diálogo con ellas; construir y discutir nuevas categorías y conceptos que permitan pensar mejor la educación, sus prácticas y sus discursos; identificar la especificidad del aporte filosófico a la mejora de la educación; facilitar la organización de grupos docentes de investigación y autoformación.

¿Qué es una buena clase de Filosofía? ¿Cuál es la especificidad de una educación filosófica? (más alla de la clase de Filosofía.) son las preguntas originales de que partimos y que comprometen toda la investigación. La intención no es, encontrar una respuesta normativa ni unívoca a estas interrogantes. Más interesante resulta hacer visibles los supuestos que las atraviesan, dilucidar cómo se traducen en el imaginario de los docentes actuales de filosofía, cuáles es el grado de coherencia o de conflicto entre lo que los profesores hacen y lo que creen que debería hacerse, cuáles son las condiciones de posibilidad para que se generen prácticas filosóficas hoy en las aulas de educación media, cuáles son los obstáculos que se reconocen como desafíos pedagógicos más acuciantes. La exigencia metodológica de centrar los contenidos y focalizar el análisis., implicó direccionar la revisión del material en el sentido de identificar, en los discursos y en las prácticas de los docentes, cuestiones recurrentes y potentes para la reflexión didáctico-filosófica. En ese sentido, se seleccionaron tres categorías: rigor filosófico, interlocutor filosófico y texto filosófico. Las categorías se pueden traducir en estos problemas:

¿Qué se entiende por rigor filosófico? ¿̇Es asimilable al rigor científico? En nombre del rigor, ¿̇no se corre el riesgo de "normalizar" la filosofía, socavando la condición de "mala conciencia de su tiempo"?

¿Qué significa que tenga lugar lo filosófico en el aula? ¿Quiénes pueden ser interlocutores del diálogo filosófico? ¿Puede suceder hoy el encuentro filosófico en las aulas de educación media? ¿Cómo reconoce el profesor al alumno como potencial interlocutor? El docente, ¿̇muestra siempre disposición para generar un espacio filosófico en el aula? ¿̇Se trata de descubrir al buen interlocutor o de provocarlo como acontecimiento a través de buenas estrategias? Cuáles son los obstáculos? ¿Cuáles son las condiciones de posibilidad? 
¿Qué es un texto filosófico? Qué es una lectura filosófica de un texto? ¿Qué es un texto?

En la tercera investigación sobre Argumentación se consideraron distintas corrientes en Teoría de la Argumentación, diversas reflexiones sobre el lugar de la discusión en el aula y múltiples investigaciones sobre la enseñanza y el aprendizaje de la argumentación.Partimos de algunas convicciones:

- el valor de la enseñanza de la argumentación en los estudiantes consiste en desarrollar habilidades para leer críticamente la sociedad, para interpretar los diversos discursos sociales y para insertarse en los espacios de debate y discusión públicos con capacidad de fundamentar o refutar opiniones, construir y defender las propias posturas.

-el interés por evitar el abordaje instrumental de la enseñanza de la argumentación y la reducción del buen pensar a reglas formales.

- la preocupación por evaluar la fortaleza o debilidad de los argumentos en función del contexto en que se despliegan los discursos.

- la exigencia de combinar el abordaje sintáctico de la argumentación con la exploración semántica, con los contenidos, tomando en cuenta el atravesamiento filosófico e ideológico de los discursos.

- el valor de la enseñanza y el aprendizaje de la argumentación en el ámbito educativo formal y la posibilidad de que las capacidades ejercitadas en ese ámbito puedan ser transferidas por los estudiantes a otros espacios de la vida social.

Sobre esas bases se hicieron opciones y se eligieron algunas referencias que se consideran especialmente significativas para esta investigación. Como concepciones sobre la argumentación se optó por la pragma-dialéctica y el modelo dialogal en Plantin. Recurrimos a Carlos Pereda para pensar las relaciones entre argumentación y violencia y a Pascal Michon para interpretar los debates desde la perspectiva del ritmo. Respecto al concepto, elegimos planteos sostenidas por Kusch, Deleuze y Guattari y Cruz. Tuvimos en cuenta muy especialmente los aportes de Vaz Ferreira y Tozzi sobre el valor y las condiciones de la discusión en clase. Rastramos antecedentes de investigaciones sobre argumentación y 
educación, centrando la búsqueda en experiencias recientes en América Latina. A partir del marco conceptual, se elaboraron criterios a tener en cuenta en la interpretación, análisis y valoración de los discursos registrados. En la investigación de campo trabajamos en 6 grupos de $2^{\circ}$ año de Bachillerato en distintas instituciones, cuatro públicas y dos privadas, con perfil sociocultural diferente, con 12 profesores, 6 de Biología y 6 de Filosofía, y con 100 alumnos.

Observamos la primera clase de Filosofía y una clase de Biología en cada grupo. Hicimos 2 intervenciones de investigación en el espacio de Filosofía, una al comienzo del curso y otra sobre el final.

En la primera intervención se seleccionó una cuestión polémica, que estaba siendo efectivamente discutida en nuestro país a nivel político y social: "el reconocimiento legal del matrimonio igualitario" (que fue aprobada ese mismo año por el Parlamento).

La segunda intervención se centró en un problema de Bioética y se propuso la cuestión del "consentimiento informado", considerando que permitía recoger elementos de los procesos de enseñanza y aprendizaje de las habilidades argumentativas que pudieran haberse desarrollado en las dos asignaturas que tomamos como base para el desarrollo de la investigación: Filosofía y Biología. Registramos la producción individual de discursos argumentativos por escrito de todos los alumnos y la producción oral en los dos debates en cada clase.

En cuanto a las referencias filosóficas son múltiples. Una fuente privilegiada son las reflexiones sobre la educación y la enseñanza de la Filosofía del filósofo uruguayo Carlos Vaz Ferreira.

Trabajamos también en dialogo con varios colegas latinoamericanos como Alejandro Cerletti, Walter Kohan, Silvio Gallo...

Tomamos en cuenta los aportes de Michel Tozzi sobre enseñanza de la filosofía y el lugar de la discusión en las clases y de Jorge Larrosa para pensar el vínculo entre experiencia y educación.

Me interesa especialmente pensar desde Foucault para considerar las prácticas pedagógicas donde no sólo circulen saberes sino que provoquen en los educandos 
y docentes cambios en la experiencia de sí. Creemos que la educación filosófica puede aportar a que los sujetos reconozcan cómo han entrado a formar parte de una determinada representación de la verdad, cuáles son las reglas de juego de los dispositivos que han construido su subjetividad y percibir también la articulación saber-poder.

Resultan muy interesantes las observaciones de Lyotard, en Memorial a propósito del curso filosófico, en La posmodernidad (explicada a los niños).

Y en general, muchos clásicos de la Historia de la Filosofía pueden reinterpretarse para pensar la educación filosófica en la actualidad.

Elisete: Em sua perspectiva, quais são os desafios que estão colocados para a escola pública e para o ensino de Filosofia no Uruguai, atualmente?

Marisa: Reconocemos algunos obstáculos. Por un lado subsisten en nuestros profesores de enseñanza media, prácticas docentes rutinizadas, inerciales, ancladas en cierta sensación de malestar permanente, abonadas por una modalidad de trabajo aislado, solitario. Esta situación, bastante generalizada, responde a diversas causas, algunas de las cuales están ligadas a las condiciones del ejercicio de la docencia (baja remuneración, multiempleo, burocratización de tareas). Por eso, vemos la necesidad de colectivizar las experiencias dispersas de los profesores; de interpelar los supuestos de prácticas de enseñanza y sus imaginarios; de promover la discusión explícita de las experiencias exitosas o no; de pensar y repensar obstáculos y condiciones de posibilidad de acciones pedagógicas deseables; de fomentar una cultura de la creación que no se agote en iniciativas aisladas y se comprometa colectivamente en pensar la cuestión de la enseñanza como problema filosófico. Por otra parte, la Educación está siendo interpelada por una profunda y novedosa diversidad de sus educandos, frente a la cual sólo se exploran tímidas estrategias. Proponemos comprender esta diversidad como multiculturalidad. Es decir, el sistema educativo tiene que vérselas con una gama amplísima de subculturas que coexisten en sus aulas, subculturas que no responden en nuestro país a variables étnicas, sino socioculturales, socio- económicas, socio- 
etarias: culturas de la pobreza, culturas de la abundancia, tribus urbanas, etc. Hoy esa diversidad opera fundamentalmente como generadora de violencia, o de prácticas de encapsulamiento fragmentado. Por este camino lo que aparece claro es un profundo quiebre de la comunidad, es decir la imposibilidad de compartir lo común. Este escenario social ingresa a las instituciones educativas y las desacomoda fuertemente. El debilitamiento del sentido de comunidad tiene efectos significativos en el vínculo pedagógico. El deseo de aprender parece circular cada vez con menos fuerza en las aulas de adolescentes. Quizás persiste el deseo de saber, pero sin que lo acompañe la disposición para el esfuerzo que implica aprender. Se multiplican las resistencias.. La cuestión es que muchos alumnos no pueden o no quieren participar en el juego pedagógico. Las instituciones educativas, cuyo reconocimiento en el imaginario social atraviesa una profunda crisis, frecuentemente responden con formaciones reactivas rigidizando su formato tradicional para asegurarse que "la vida" no entre a la escuela y no la jaquee. Muchos docentes reproducen inercialmente rutinas pedagógicas funcionales a los alumnos del pasado, con la fantasía de que así los estudiantes actuales mutarán en aquellos que añoran como modelos. En síntesis: fuertes negaciones, intensa insatisfacción y auténtica angustia atraviesan los vínculos pedagógicos. Pero también se perciben líneas de fuga, muchas veces la creatividad y el compromiso se concretan en experiencias en las que estudiantes y docentes recuperan el deseo pedagógico y la capacidad de disfrute en el encuentro.

Prof. Marisa Berttolini

\section{Currículo de Marisa Berttolini}

Profesora egresada del IPA en la especialidad Filosofía.

Exinspectora nacional de la asignatura Filosofía. Consejo de Educación Secundaria. Uruguay.

Docente de Didáctica de la Filosofía. Instituto de Profesores Artigas.

Docente de Epistemología, CLAEH. Maestría en Docencia de la Educación Media. Investigadora en el área de Educación Filosófica en la Enseñanza Media. 
Publicaciones Libros:

Diversidad Cultural e Interculturalidad. Ediciones Novedades Educativas. Buenos Aires. 2009. En coautoría con Prof. Mauricio Langon.

Materiales para la Construcción de Cursos de Filosofía. "¿Qué es Filosofía?"AZ Editora. Montevideo. 1997. En coautoría con Prof. Langon y Profa. Quintela.

Participación como ponente en encuentros nacionales e internacionales.

Publicación de artículos en libros y revistas especializadas en la enseñanza de la filosofía.

Vicepresidenta de la Asocicación de Filosofía del Uruguay. 